\title{
Análisis Del Estrés Laboral Y Su Repercusión En La Salud Física Y Mental En Operadores De Tracto Camión
}

\author{
Homero Janai López-Trejo, (MAE) \\ Colegio Interdisciplinario de Especialización. Puebla, México \\ Universidad Popular Autónoma de Veracruz \\ Claudia Olivia Carrera-Salazar, (MI) \\ María Isela Eurrieta-Ortiz, (MBA) \\ Luz del Carmen García-Arroyo, (MI) \\ Colegio Interdisciplinario de Especialización. Puebla, México \\ Montserrat Gómez-Márquez, (MF) \\ Jesús Gerardo LLanillo-Navales, (MTE) \\ Colegio Interdisciplinario de Especialización. Puebla, México \\ Instituto Tecnológico Superior de Huatusco \\ Martha Marín-Ramos, (MTE) \\ Colegio Interdisciplinario de Especialización. Puebla, México \\ Telebachillerato Comunitario del Estado de Veracruz \\ Fernando Agustín Romo-Celis, (MAE) \\ Colegio Interdisciplinario de Especialización. Puebla, México \\ Universidad Politécnica de Huatusco \\ Manuel González-Pérez, (PhD) \\ Colegio Interdisciplinario de Especialización. Puebla, México \\ Sistema Nacional de Investigadores (nivel 1)
}

Doi: 10.19044/esj.2018.v14n11p10 URL:http://dx.doi.org/10.19044/esj.2018.v14n11p10

\begin{abstract}
The Work stress (WS) is one of the leading causes of deterioration of physical and psychological well-being within organizations. In this research the multivariate dependence of some signs and symptoms of work-related illness was analyzed and calculated. A cross-sectional and descriptive study of a base of logistic operations of fleet dedicated to the transportation of beer in the center of the state of Veracruz was carried out. The population studied was 230 operators. Only male operators, active within the company, were included and operators who at the time of the study were under medical and vacation disability were excluded. The multivariate Pearson correlation was used to validate the research questions and chi-square to calculate the
\end{abstract}


dependence of variables. As a result, we obtained five variables taken from two in two that were dependent on each other. We conclude that there is a healthy relationship between the observed variables, which are listed in order of pathological impact and their physiological reexaminations in the organism. The pressure within work caused by job stress represents a potent source of mental illness such as depression.

Keywords: Labor stress, Physical health, Mental health, Operators of tract truck

\section{Resumen}

El estrés laboral (E.L.) es una de las principales causas de deterioro del bienestar físico y psicológico dentro de las organizaciones. En esta investigación se analizó y calculó la dependencia multivariada de algunos signos y síntomas que son provocados por estrés laboral. Se realizó un estudio transversal y descriptivo de una base de operaciones logísticas de flota dedicada a la transportación de cerveza, La población estudiada fue de 230 operadores. Se incluyeron únicamente operadores varones, activos dentro de la empresa y se excluyó a los operadores que en el momento del estudio se encontraban bajo incapacidad médica y de vacaciones. Se utilizó la correlación de Pearson multivariada para validar las preguntas de investigación y chi cuadrada para calcular la dependencia de variables. Como resultado obtuvimos cinco variables tomadas de dos en dos que resultaron dependientes una de otra. Concluímos que existe una fuerte relación entre las variables observadas, las cuales se enlistan en orden de impacto patológico y sus repercusiones fisiológicas en el organismo. La presión dentro del trabajo causado por el estrés laboral representa una fuerte fuente de enfermedades mentales tales como la depresión.

Palabras claves: Estrés laboral, Salud física, Salud mental, Operadores de tracto-camión

\section{Introducción}

El estrés laboral (E.L.) es una de las principales causas de deterioro del bienestar físico y psicológico dentro de las organizaciones y es un elemento empobrecedor de la salud (Del Hoyo, 2004) y la eficiencia tanto individual como colectiva (Castro-Ruiz, 2018). Hemos de entender que el E.L. es un problema que ha ido en aumento en las últimas décadas debido a que han cambio radicalmente los esquemas de trabajo: de fuerza y trabajo muscular se ha añadido esfuerzo mental y reducción de tiempos por la implementación de tecnología en muchos de los procesos (Sanz, 2017; Aranzaes, 2008). 
En México, los operadores de tracto camión (OTC), han sido por mucho considerados como un oficio de segunda (Sanz, 2017), hasta cierto punto menospreciado por los bajos requisitos académicos que son solicitados por las autoridades tales como, solo saber leer y escribir, sin embargo, se piden cerca de 190 horas de capacitación (36\% teoría y 64\% practica) por lo que debe ser considerado como un trabajo especializado y donde además el OTC se expone a riesgos y exigencias que no solo se limitan a las de su organización sino a las propias que participan dentro de la división técnica del transporte; las largas distancias, jornadas extenuantes, ritmo intenso y trabajo nocturno, las condiciones climatológicas y de carreteras, inseguridad, son solo algunos de los estresores a los que hacen frente los OTC y que pueden afectar la salud física y mental de los operadores.

El Instituto Mexicano del Seguro Social (IMSS) define al estrés como un "conjunto de reacciones físicas y mentales que la persona sufre cuando se ve sometida a diversos factores externos que superan su capacidad para enfrentarse a ello". Y cuando éstas son consecuencias de las crecientes demandas cotidianas en el desempeño de su trabajo toma el nombre de "E.L.", estas consecuencias no solo afectan la salud del trabajador (Del Hoyo, 2004), sino también su entorno más próximo ya que genera un desequilibrio entre lo laboral y lo personal. El E.L. es catalogado como una enfermedad (Miranda, 2018; Aguilar, 2018) y por tal motivo tienen signos y síntomas tales como: ansiedad, miedo, irritabilidad, mal humor, frustración, agotamiento, impotencia, inseguridad, desmotivación, intolerancia, dolor de cabeza, problemas de espalda o cuello, malestar estomacal, fatiga, infecciones, palpitaciones, respiración agitada, aumento de la presión sanguínea, agotamiento, mayor riesgo de obesidad y de problemas cardiovasculares, deterioro en la memoria, problemas de sueño, etc., que pueden traer consigo consecuencias a largo plazo como: reducción de productividad, descenso en la calidad de vida, problemas de salud física y/o mental, trastornos de depresión y ansiedad, problemas familiares, riesgos de alcoholismo y otras adicciones (Sanz, 2017; Vieco, 2014; De Camargo, 2010), etc., por lo que debe ser tratado con seriedad por un profesional de la salud.

Los estudios en México, son en su gran mayoría sobre choferes del sector urbano y en el sector de transporte de pasajeros, los OTC representan el $49 \%$ de todo el empleo en el sector transporte en México, por lo que son parte estratégica en la cadena de suministros (Sanz, 2017).

\section{Antecedentes}

El estrés lo hemos entendido en la mayoría de los casos como un proceso adaptativo a algún evento adverso o perturbador que provoca una exigencia mayor en el rendimiento normal de la persona o trabajador (Del Hoyo, 2004; De Camargo, 2010; Romero, 2004), el cual puede ser percibido 
por el cuerpo humano como un estímulo sistémico (sepsis, lesión tisular, exposición a temperaturas altas o abatidas, etc.) o estímulo psicológico (mentales o emocionales) (Romero, 2004). Diariamente nos desarrollamos en medio de situaciones estresantes, es parte natural de nuestra vida, por lo que es una respuesta funcional de nuestro cuerpo, la cual nos prepara para actuar o escapar ante posibles amenazas (Del Hoyo, 2004; Castro, 2018; Aguilar, 2018; Durán, 2004; Lima, 2003). Quedando en el entendido que existen estímulos estresantes o estresores y respuestas fisiológicas a esos estímulos, que cuando éstos se mantienen por un periodo prolongado se vuelven nocivos y generan la aparición de alteraciones indeseables (De Camargo, 2010; Silvia, 2004).

A través de diversas evidencias científicas hoy sabemos que el estrés es un proceso fisicoquímico o emocional inductor de una tensión y que provoca una comunicación constante y bidireccional entre el sistema neuroendócrino y la respuesta inmunológica a lo que hoy conocemos como "interacciones neuroendocrinoinmunológicas" (NEI) (Romero, 2004; López, 2008); las que favorecen la liberación de: a) las citocinas proinflamatorias, b) la hormona liberadora de corticotrofina (CRH) y c) el cortisol, así como de un amplio grupo de neurotransmisores que inducen, en su conjunto, la aparición de alteraciones conductuales (Aguilar, 2018; Romero, 2004; Silvia, 2004; López, 2008). Ante la presencia de un evento estresante el cuerpo humano activa el funcionamiento de tres ejes fisiológicos, el eje neural, el neuroendocrino y el eje endocrino. En donde el eje neural provoca el aumento del tono muscular, la paralización corporal, la descarga masiva del simpático y la reacción de alerta cortical. El eje neuroendocrino inicia con la secreción de adrenalina y noradrenalina por la médula adrenal y sus consecuencias corporales. El eje endocrino inicia la activación del eje funcional hipotálamohipófisis-corteza suprarrenal-cortisol (De Camargo, 2010; Lima, 2003; Silvia, 2004). Esto inicia una respuesta adaptativa ante los estímulos estresantes que consiste en una elevación de los niveles circulantes de citocinas proinflamatorias (IL-1, IL-6) - factor de necrosis tumoral alfa (TNF- $\alpha$ ), interleucina IL-6 e IL-1, producidas por las células de la respuesta inmunológica, como los linfocitos y macrófagos. Al alcanzar una concentración de 10nM, estas citocinas proinflamatorias son capaces de unirse a sus receptores y estimular al Sistema Nervioso Central (SNC) (Romero, 2004; Silvia, 2004) Fig.1. 


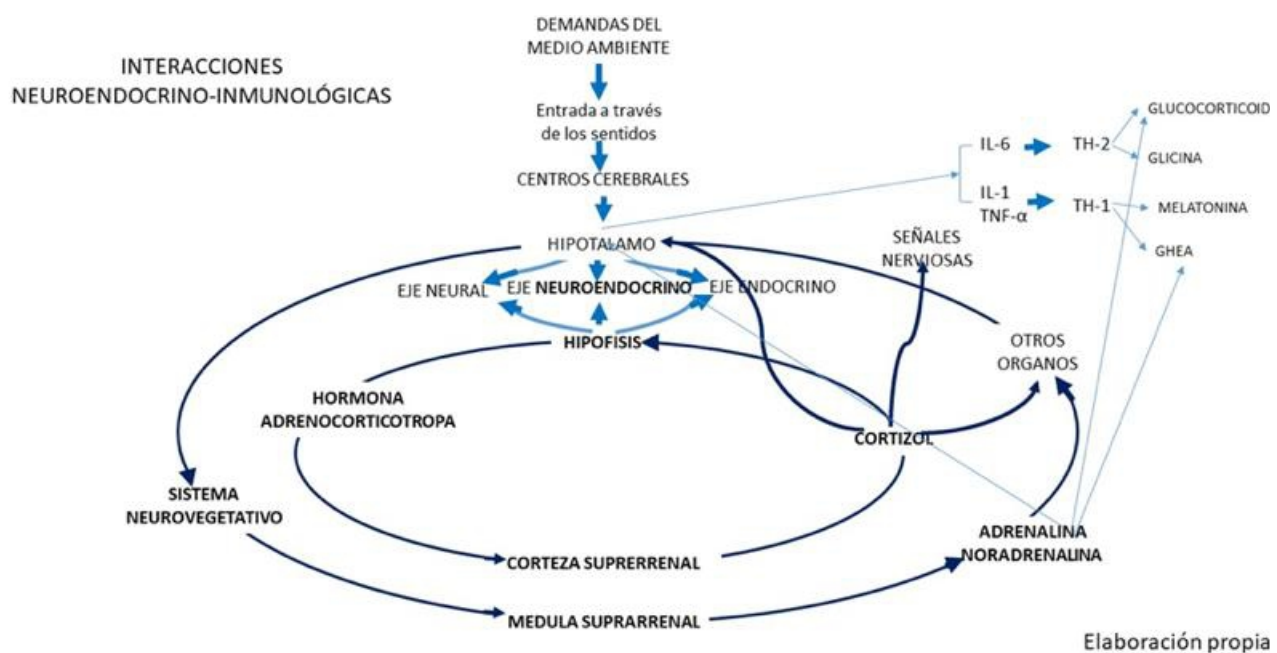

Figura 1. Interacciones Neuroendocrinoinmunológicas.

Los procesos NEI son los encargados de poner el cuerpo en estado de alerta preparatoria, el cual consiste en una serie de reacciones fisiológicas que preparan al sujeto para pelear o huir. Esto permite enlazar el fenómeno del estrés con los fenómenos psicofisiológicos de la emoción. En ambos intervienen en los siguientes procesos (Del Hoyo, 2004, Silvia, 2004):

- Dilatación de las pupilas.

- Dilatación bronquial.

- Movilización de los ácidos grasos, pudiendo dar lugar a un incremento de lípidos en sangre (posible arterioesclerosis).

- $\quad$ Aumento de la coagulación.

- Incremento del rendimiento cardíaco que puede desembocar en una hipertensión arterial.

- Vasodilatación muscular y vasoconstricción cutánea.

- Reducción de los niveles de estrógenos y testosterona, que son hormonas que estimulan el desarrollo de las características sexuales secundarias masculinas.

mamaria.

- Incremento de la producción de tiroxina, que favorece el metabolismo energético, la síntesis de proteínas.

Es evidente la existencia de un compromiso de toda la economía del organismo.

Hans Selye, investigador alemán, mejor conocido como padre del estrés, gracias a las aportaciones que hizo por sus investigaciones en 1936 y que dieron lugar al llamado síndrome general de adaptación (SGA) (De 
Camargo, 2010; Silvia, 2004), estableció que: "algo de estrés es esencial y saludable; la cantidad óptima de estrés es ideal, pero demasiado estrés es dañino". Por lo tanto, una pequeña cantidad de estrés es necesario (De Camargo, 2010; Silvia, 2004; Romero, 2018; Mafud, 2017). Dentro de sus investigaciones estableció las fases del estrés o SGA, las cuales son: 1. Fase de Alarma, 2. Fase de Resistencia, 3. Fase de Agotamiento, donde primero es percibido e identificado el estímulo por parte de nuestro organismo; al cual inicialmente responde nuestro Sistema Nervioso y después se expresa el Sistema Endocrino (De Camargo, 2010).

El agente estresor, es todo aquel estímulo que provoca la respuesta al estrés. Puesto que casi cualquier cosa puede convertirse en un estresor (Aranzaes, 2008; Cárdenas, 2015; Quiroz, 2016) sería imposible elaborar una lista exhaustiva de estresores. Sin embargo, se puede afirmar que algunas condiciones externas tienen mucha mayor probabilidad de operar como estresores que otras, en el entendido que todo es parte del medio ambiente (MA). Algunos aspectos del MA son físicos, sociológicos y otros psicológicos. Desde esta objetividad los factores de estrés presentes en situación de trabajo se pueden clasificar en tres grandes grupos (Del Hoyo, 2004; Castro-Ruiz, 2018; De Camargo, 2010):

1. Estresores del ambiente físico: Condiciones físicas de carretera, condiciones ambientales, temperatura, tráfico.

2. Estresores relativos al contenido de la tarea: Carga mental, control sobre la tarea, tiempos de entrega, etc.

3. Estresores relativos a la organización: Conflicto y ambigüedad del rol jornada de trabajo, relaciones interpersonales, promoción y desarrollo de la carrera profesional

Existen diferencias individuales (situaciones particulares vividas, expectativas, experiencias pasadas, actitudes, aptitudes y aun sus propios sentimientos)( Del Hoyo, 2004; Mansilla, 2004), que ante la presencia de un estresor pueden dar un desequilibrio que conduzca al estrés (Lima, 2003; Moscoso, 2015). Estas diferencias individuales también pueden variar en función de diversos factores tales como: edad, necesidades, expectativas, estado de salud, etc., es por ello que durante la gestación del E.L. Será determinante conocer rasgos de estas diferencias individuales para determinar algún grado de afectación en el trabajador (Vieco, 2014; Moreno, 2011).

En el área laboral, el OTC en México puede definirse como una actividad u oficio especializado, predominantemente masculino, catalogado como un trabajo no clásico (Sanz, 2017), asociado a un esquema de servicios que no sólo depende del aprendizaje o del esfuerzo físico y mental, si no que exige al operador, la necesidad de desarrollar diversas habilidades y capacidades humanas que dependerán en mayor medida de la experiencia adquirida y la subjetividad del OTC (Sanz, 2017; Cárdenas, 2015). 
Los OTC tienen un estilo de vida muy característico, que incluyen malos hábitos de alimentación, alta prevalencia de consumo tabaco, alcohol y drogas, gran exigencia física y mental, horarios irregulares, tanto de trabajo como de horas de sueño y falta de actividad física. Es por ello que los OTC son susceptibles a gran cantidad de problemas de salud, que incluyen obesidad, enfermedades cardiovasculares, trastornos metabólicos, enfermedades musculo-esqueléticas (Fernández, 2012; Henao, 2016), estrés ocupacional y enfermedades psicosociales (Henao, 2016; Fernández, 2010). Aun con todas estas similitudes no son grupos homogéneos, las condiciones varían de acuerdo al tipo de vehículo, el producto que transportan, la distancia que recorren, la regulación laboral y la protección social de acuerdo al territorio donde laboran.

Dentro de la población estudiada los OTC se encuentran divididos en 2 grupos, los operadores locales o "movedores" encargados de transportar el producto de la fábrica a la base de operaciones con un horario de 8 horas en 3 turnos y los operadores de carretera que son los encargados de llevar el producto a los distintos CEDIS o Agencias en la República Mexicana; dentro de este grupo existe una subdivisión a lo que llamaremos operadores de "viajes cortos" que son aquellos viajes menores a 12 horas en dar la vuelta (salida de la base, llegada al CEDIS, descarga y regreso a la base) y operadores de "viajes largos" que son aquellos con un recorrido mayor a las 12 horas por vuelta.

\section{Metodología}

Se realizó un estudio transversal y descriptivo donde se analizó el estrés ocupacional de una base de operaciones logísticas de flota dedicada a la transportación de cerveza, con una población estudiada de 230 operadores donde se incluyeron únicamente operadores varones, activos dentro de la empresa y se excluyó a los operadores que en el momento del estudio se encontraban bajo incapacidad médica y de descanso.

La población de OTC estudiada oscila entre los 22 años como mínimo y los 58 años de edad cumplidos al momento de realizado el estudio, con una media de 41.6 años y con una antigüedad en años completos dentro de la empresa que va desde los 0 hasta los 33 años, con una media de 12 años de antigüedad.

\section{Instrumentos de medición}

Para poder determinar el grado de E.L. de los OTC se hizo uso de un cuestionario tipo encuesta, donde los items a evaluar fueron los más frecuentes signos y síntomas causados por el estrés laboral y donde se dio una ponderación de 1 a 5 siendo 1 totalmente sano y 5 datos manifiestos de la enfermedad para cada cuestionamiento, se incluyó una escala de valor general 
donde incluía al OTC dentro de un grado de estrés laboral. Las preguntas se sometieron a escrutinio por correlación de Pearson donde se verificaron los ítems para determinar si existe alguna confusión en la redacción, el cuestionario se aplicó por igual a la población estudiada sin distinción a que grupo de operador pertenecía.

\section{Resultados y discusión}

Validación del instrumento con correlación de Pearson.

En la tabla 1 se puede ver la correlación de Pearson. Esta correlación nos muestra que no existe repetición alguna en las preguntas. Una vez realizada esta validación, se aplicó a 230 trabajadores.

Tabla 1. Validación por correlación de Pearson

\begin{tabular}{ccccccccccc}
\hline & Pre 1 & Pre 2 & Pre 3 & Pre 4 & Pre 5 & Pre 6 & Pre 7 & Pre 8 & Pre 9 & Pre 10 \\
\hline Pre 1 & 1.000 & & & & & & & & & \\
Pre 2 & 0.447 & 1.000 & & & & & & & & \\
Pre 3 & 0.520 & 0.448 & 1.000 & & & & & & & \\
Pre 4 & 0.264 & 0.359 & 0.357 & 1.000 & & & & & & \\
Pre 5 & 0.306 & 0.225 & 0.311 & 0.208 & 1.000 & & & & & \\
Pre 6 & 0.332 & 0.279 & 0.340 & 0.337 & 0.316 & 1.000 & & & & \\
Pre 7 & 0.386 & 0.485 & 0.461 & 0.460 & 0.306 & 0.428 & 1.000 & & & \\
Pre 8 & 0.483 & 0.361 & 0.460 & 0.278 & 0.192 & 0.348 & 0.420 & 1.000 & & \\
Pre 9 & 0.205 & 0.171 & 0.177 & 0.225 & 0.207 & 0.166 & 0.143 & 0.180 & 1.000 & \\
Pre 10 & 0.231 & 0.356 & 0.190 & 0.134 & 0.151 & 0.237 & 0.319 & 0.240 & 0.136 & 1.000 \\
\hline
\end{tabular}

\section{Dependencia de variables}

A continuación, se aplicó la prueba de chi cuadrada para hallar la relación de variables de dos en dos (representada por cada ítem)

La tabla 1 nos muestra los resultados de los valores crudos tomados directamente de la encuesta. En la tabla 2 obtuvimos los valores esperados y en la tabla 3 se muestran los valores estadísticos de la chi cuadrada. 7.

En el caso específico que se muestra, se compararon las preguntas $8 \mathrm{y}$

8) Siento mucha presión en mi trabajo.

7) Me siento de mal humor.

Tabla 2. Tabla de contingencia preguntas 8 y 7 .

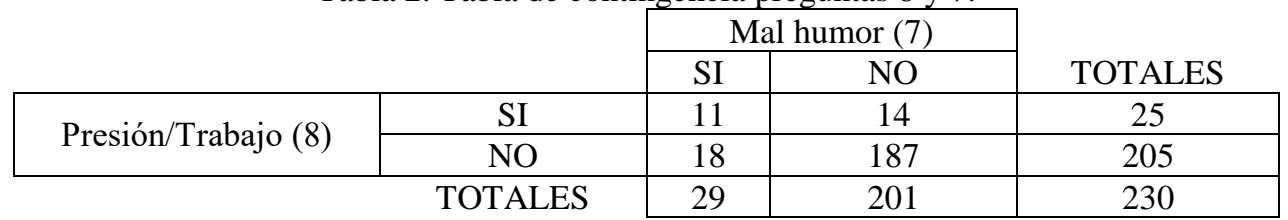


Tabla 3. Tabla de valores esperados preguntas 8 y 7 .

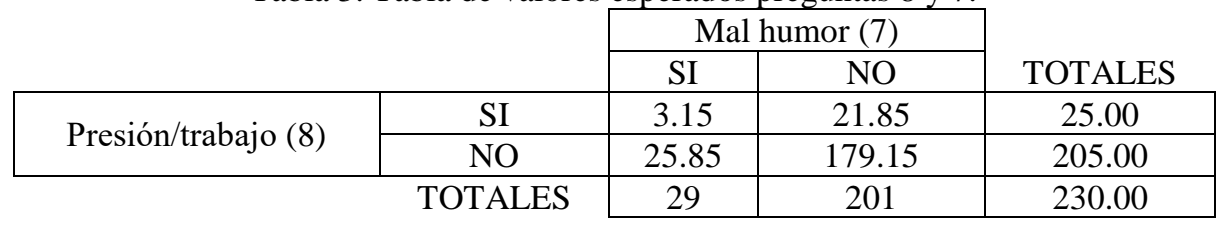

Tabla 4. Tabla de valores estadísticos preguntas 8 y 7 .

\begin{tabular}{|c|c|c|c|c|}
\hline & \multirow{2}{*}{\multicolumn{2}{|c|}{ Mal humor (7) }} & \multirow[b]{3}{*}{ TOTALES } \\
\hline & & & & \\
\hline & & SI & $\mathrm{NO}$ & \\
\hline \multirow{2}{*}{ Presión/trabajo (8) } & SI & 19.54 & 2.82 & 22.36 \\
\hline & $\mathrm{NO}$ & 2.38 & 0.34 & 2.73 \\
\hline & TOTALES & 21.92 & 3.16 & 25.08 \\
\hline & & & & 3.84 \\
\hline
\end{tabular}

El resultado de chi cuadrada observada o experimental nos arroja un valor de 25.08 (tabla 4); mientras que el valor de chi cuadrada teórica nos muestra un valor de 3.84. Se puede deducir entonces que las variables 7 y 8 son estadísticamente dependientes. En las figuras 2 y 3 se ejemplifica mejor esta dependencia. Podemos observar que la chi calculada está muy lejos de la chi teórica y fuera de la curva normal de Pearson para 1 grado de libertad. Se interpreta como un $\mathrm{p}$ valor casi de cero para la dependencia; 1 para la independencia.

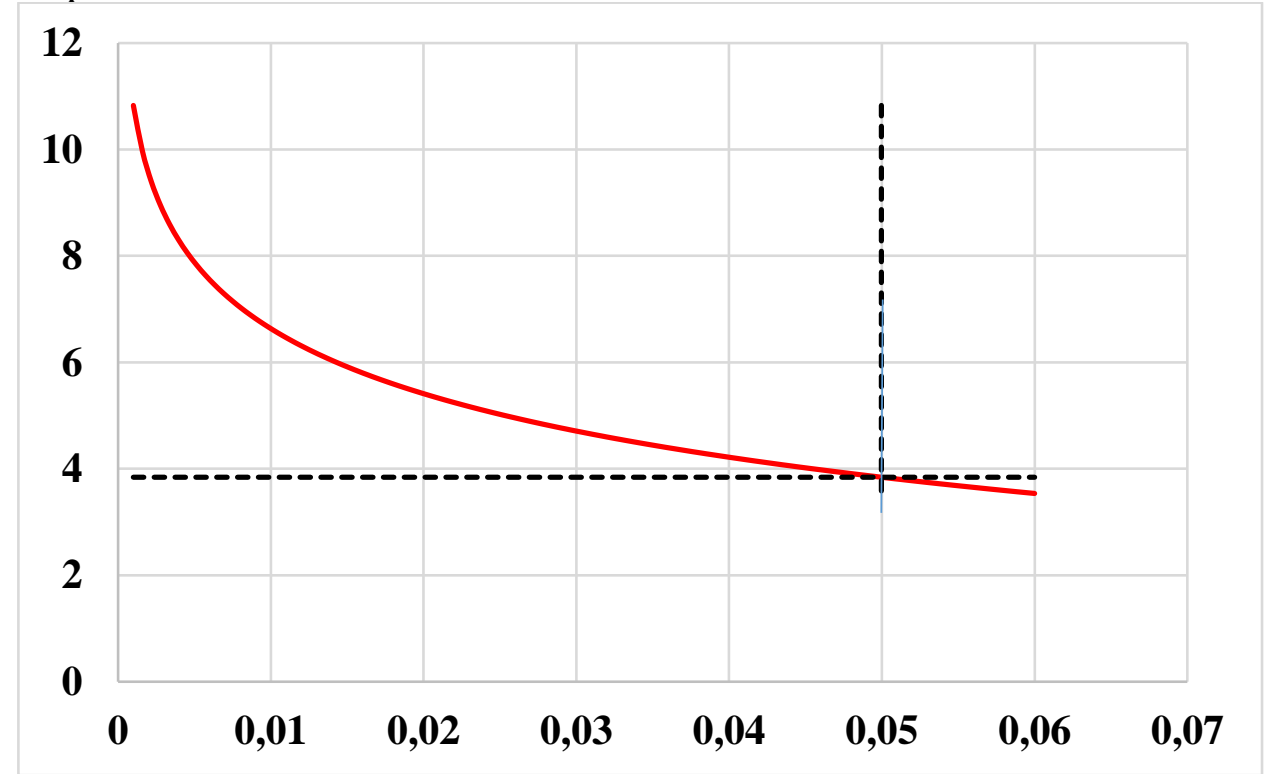

Figura 2. Gráfica de la función inversa teórica: $\mathrm{p}$ valor vs chi cuadrada de 1 grado de libertad. Se observa la intersección $(0.05,3.08)$ para chi teórica. $95 \%$ de confianza para la independencia, $5 \%$ de confianza para la dependencia. 


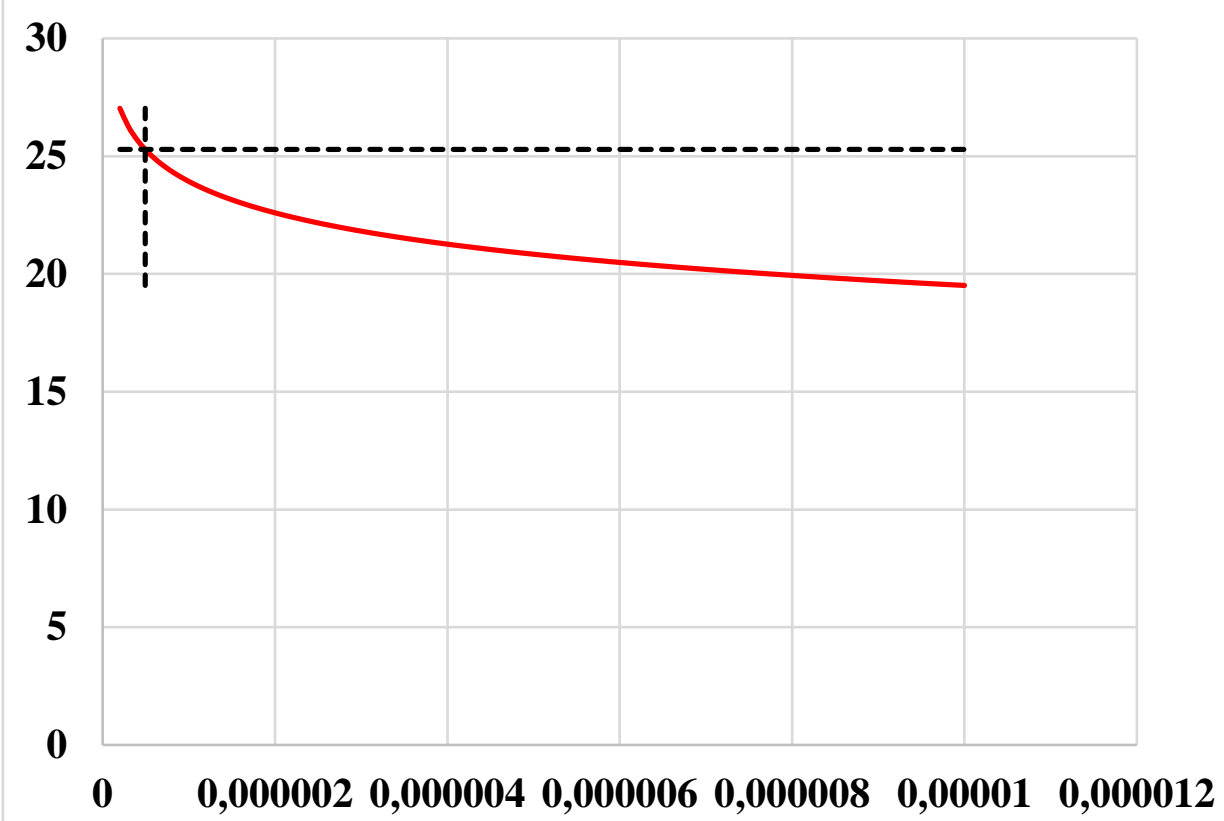

Figura 3. Gráfica de la función inversa teórica: $\mathrm{p}$ valor vs chi cuadrada de 1 grado de libertad. Se observa la intersección $(0.000000494,25.28710621)$ para chi observada o calculada. Casi $100 \%$ de confianza para la dependencia, $0 \%$ de confianza para la independencia. tabla 5 .

Otras comparaciones que resultaron dependientes, se muestran en la Tabla 5. Tabla de variables dependientes calculadas por chi cuadrada.

\begin{tabular}{|c|c|c|c|c|}
\hline $\begin{array}{c}\text { Variable } \\
\text { independiente }\end{array}$ & $\begin{array}{c}\text { Variable } \\
\text { dependiente }\end{array}$ & $\begin{array}{c}\text { Chi observada } \\
\text { o calculada }\end{array}$ & $\begin{array}{c}\text { Chi teórica } \\
\text { P valor }(0.05)\end{array}$ & Observación \\
\hline Me enfermo (9) & $\begin{array}{l}\text { Me duele la } \\
\text { cabeza (6) }\end{array}$ & 6.06 & 3.84 & $\begin{array}{c}\text { Variables } \\
\text { dependientes }\end{array}$ \\
\hline $\begin{array}{l}\text { Se me dificulta } \\
\text { dormir (4) }\end{array}$ & $\begin{array}{c}\text { Aunque } \\
\text { duerma me } \\
\text { siento fatigado } \\
(1)\end{array}$ & 7.45 & 3.84 & $\begin{array}{c}\text { Variables } \\
\text { dependientes }\end{array}$ \\
\hline$\frac{\frac{\text { Siento mucha }}{\text { presión en mi }}}{\underline{\text { trabajo (8) }}}$ & $\begin{array}{l}\text { Me duele la } \\
\text { cabeza (6) }\end{array}$ & 11.30 & 3.84 & $\begin{array}{c}\text { Variables } \\
\text { dependientes }\end{array}$ \\
\hline $\begin{array}{l}\text { Siento mucha } \\
\text { presión en mi } \\
\text { trabajo (8) }\end{array}$ & $\begin{array}{c}\text { Me siento } \\
\text { deprimido (2) }\end{array}$ & 23.98 & 3.84 & $\begin{array}{c}\text { Variables } \\
\text { dependientes }\end{array}$ \\
\hline
\end{tabular}

\section{Conclusion}

Se encuestaron 230 operadores de tracto camión y...:

1. Validamos el cuestionario usando la correlación multiple de Pearson (Tabla 1). Esto se hizo para detectar items repetidos por diferentes causas. 
2. Existe una fuerte relación entre las variables observadas, las cuales se enlistan en orden de impacto patológico y sus repecusiones fisiologicas en el organismo (tabla 5).

3. La presión dentro del trabajo causado por el estres laboral representa una fuerte fuente de enfermedades mentales tales como la depresión, segun se observa en la tabla 5.

4. El estrés laboral es causa directa de enfermedades físicas tales como cefalea, gastritis y ausencia de sueño (tabla 5).

5. Las enfermedades mentales y físicas causadas por el estrés ocupacional (tabla 5) pudieran ser una causa del incremento de ausentismo laboral.

6. El mal humor en el operador de tracto camión puede ser una fuerte consecuencia de estrés ocupacional al cual esta siendo sometido (tablas 2, 3, $4)$.

\section{References:}

1. Del Hoyo, M.A. Estrés laboral "documentos divulgativos", Ministerio del trabajo y asuntos sociales, I.N.S.H.T. MADRID, 2004

2. Castro-Ruiz, A., Castro-Muñoz, J. R., De La Fuente-Pérez, C. S., \& Pérez-Shiels, I. (2018). Nivel de estrés y sus efectos en el agotamiento, despersonalización e insatisfacción al logro. MULTIDISCIPLINARY HEALTH RESEARCH, 2(3).

3. Sanz, L. D. B. (2017). Choferes del autotransporte de carga en México: investigaciones sobre condiciones laborales y la cadena de suministro. Revista Transporte y Territorio, (17), 251-266.

4. Aranzaes, C., \& García, A. (2008). Un estudio exploratorio sobre estresores laborales en conductores de transporte público colectivo en el Estado de Morelos, México. Recuperado el, 7.

5. Miranda-Ledesma, A., \& Batista-Anache, S. (2018). Estrés: elemento clave en la formación laboral para la salud ocupacional. Maestro y Sociedad, 1(2), 67-77.

6. Aguilar, M. E. B. (2018). El estrés y su influencia en la calidad de vida. MULTIMED Revista Médica Granma, 21(6).

7. Vieco Gómez, G. F., \& Abello Llanos, R. (2014). Factores psicosociales de origen laboral, estrés y morbilidad en el mundo. Psicología desde el Caribe, 31(2), 354-385

8. De Camargo, B. (2010). Estrés, síndrome general de adaptación o reacción general de alarma. Revista medico científica, 17(2), 78-86.

9. Romero, L. P., Hernández, M. E., Salinas, F. L., \& López, G. S. (2004). Interacciones neuroendocrinoinmunológicas. Salud mental, 27(3), 1925. 
10. Durán, M. M. (2010). Bienestar psicológico: el estrés y la calidad de vida en el contexto laboral. Revista nacional de administración, 1(1), 71-84

11. Lima Mompó, G., Aldana Vilas, L., Casanova Sotolongo, P., Casanova Carrillo, P., Casanova Carrillo, C., \& García Gutiérrez, E. (2003). Influencia del estrés ocupacional en el proceso saludenfermedad. Revista Cubana de Medicina Militar, 32(2), 0-0.

12. Silvia N. (2004). NTP 355: Fisiología del estrés, CENTRO NACIONAL DE CONDICIONES DE TRABAJO, Ministerio del trabajo y asuntos sociales, España

13. López, S. (2008). Estrés oxidativo, metabolitos oxidados de dopamina y enfermedad de Parkinson. Rev Fac Med UNAM, 51(3).

14. Guerrero, J. A. J. Estrés, neuroinflamación y neurotransmisión. Omega, 3, 4.

15. Durán, M. M. (2010). Bienestar psicológico: el estrés y la calidad de vida en el contexto laboral. Revista nacional de administración, 1(1), 71-84.

16. Mafud, J. L. C., Arocena, F. L., \& Moreno, M. P. (2017). La autoeficacia como mediador entre el estrés laboral y el bienestar. Psicología y Salud, 27(1), 71-78.

17. Cárdenas, M., \& Stalin, E. (2015). Influencia de las condiciones laborales en el nivel de estrés de los conductores de la Coop. de Transporte Interprovincial Flota Bolivar (Bachelor's thesis, Quito: $\mathrm{UCE})$.

18. Quiroz, C. N. N., Rivera, L. O., \& Valero, C. Z. V. (2016). Relación entre Estrés Laboral y Estrés Cotidiano. Revista Electrónica de Psicología Iztacala, 19(2), 558.

19. Mansilla Izquierdo, F. (2004). El riesgo psicosocial en el trabajo: Una realidad emergente. Revista de la Asociación Española de Neuropsiquiatría, (89), 3-5.

20. Moscoso, M. S. Estres crónico y depresión.

21. Moreno Jiménez, B. (2011). Factores y riesgos laborales psicosociales: conceptualización, historia y cambios actuales. Medicina y Seguridad del trabajo, 57, 4-19.

22. Fernández-D Pool, J., Vélez, F., Brito, A., \& D Pool, C. (2012). Síntomas musculoesqueléticos en conductores de buses de una institución universitaria. Investigación Clínica, 53(2), 125-137.

23. Henao, A., \& Carlos, J. Prevalencia de síntomas osteomuculares en operadores de vehículos mecánicos del sistema integrado de transporte masivo en la ciudad de Pereira, Colombia 2016. 
24. SPA, C. O. P. S. (2012). Estrés laboral y consumo de sustancias psicoactivas (SPA) desde un enfoque de la salud ocupacional. Revista Peruana de Psicología y Trabajo Social, 1(1), 107-118.

25. Fernández, P. H. (2010). La influencia de los factores estresantes del trabajo en el rendimiento laboral. Invenio, 13(25). 